Bangladesh Journal of Neuroscience 2019; Vol. 35 (1): 14-21

\title{
Risk Factors Analysis in Various Subtypes of Ischemic Stroke According to TOAST Criteria in a Tertiary Care Hospital
}

\author{
MOHAMMAD RH ${ }^{1}$, ISLAM MR ${ }^{2}$, SHAHIDULLAH M ${ }^{3}$, RAHMAN HZ ${ }^{4}$, REFAYET CNH ${ }^{5}$, RASHID MB
}

\begin{abstract}
:
Background: Stroke is the third leading cause of death in adult population throughout the world and is the most common cause of severe adult physical disability. The aim of the study is to identify the major risk factors in various subtypes of ischemic stroke according to TOAST criteria. Methods: A Cross-sectional observational study was conducted from January, 2018 to December 2018 in the department of Neurology, Bangabandhu Sheikh Mujib Medical University, Dhaka. All the patients of first ever ischemic stroke within 14 days diagnosed by history, clinical examination and neuroimaging (CT scan of head / MRI of brain), meeting the inclusion and exclusion criteria were included in the study. Results: Present study showed that among the 52 ischemic stroke patients mean age of the respondents was $57 \pm 12.37$ years with a slightly higher male predominance. Male to female ratio was 1.2:1. Dyslipidemia 44 (84.6\%) and hypertension 37 (71.2\%) were the most common risk factors, followed by obesity and overweight 33(63.5\%), smoking 32 (61.5\%), diabetes mellitus 29 (55.8\%), family history of vascular event 27(51.9\%) and past history of vascular event 14 (26.9\%). TOAST Subtype distribution of study population was large-artery atherosclerosis 18 (34.6\%) followed by cardioembolism 11(21.2\%), small-vessel occlusion 10(19.2\%), stroke of other determined etiology 3(5.8\%), and stroke of undetermined etiology 10(19.2 $\%)$ of patients. In cardioembolic subtype significant association was found with ischemic heart disease $(P=0.001)$ and chronic rheumatic heart disease $(P=<0.001)$. Conclusion: In this study large-artery atherosclerosis was the most common subtype, followed by cardioembolism, small vessel occlusion, stroke of undetermined etiology and stroke of other determined etiology subtypes. Dyslipidemia was found to be the most common risk factor, others were HTN, diabetes and smoking. Ischemic heart disease and rheumatic heart disease were very important cause and comorbidities of cardioembolic types of ischemic stroke.
\end{abstract}

Key words: Ischemic stroke, Risk factors, TOAST criteria, Subtypes.

Introduction:

Worldwide stroke is the second most leading cause of death and the third leading cause of disability in adult ${ }^{1}$. Due to stroke was 5.7 million people died worldwide in 2005 and projected to rise to 7.8 million in year $2030^{2}$. According to GBD $2016^{3}$, Death due to stroke in South Asia has increased from $15 \%$ to $21 \%$ and the mean global lifetime risk of stroke has increased from $22.8 \%$ in 1990 to $24.9 \%$.
Stroke is defined by World Health Organization (WHO) as rapidly developing clinical signs of focal (or global) disturbance of cerebral function, with symptoms lasting 24 hours or longer or leading to death, with no apparent cause other than of non traumatic vascular origin ${ }^{4}$. The incidence of stroke varies among various countries. Over the last forty years, the stroke incidence in low- and middleincome countries has become more than doubled.

1. Dr. Rakib Hasan Mohammad, MD (Neurology) Student, Dept. of Neurology, BSMMU, Dhaka.

2. Prof Dr. Md. Rafiqul Islam, Professor \& Chairman, Dept. of Neurology, BSMMU, Dhaka.

3. Dr. Md. Shahidullah, Associate Professor, Dept. of Neurology, BSMMU, Dhaka.

4. Prof. Dr. Hasan Zahidur, Rahman, Professor, Dept. of Neurology, BSMMU, Dhaka.

5. Dr. Chowdhury Neamul Hossain Refayet Resident, Dept. of Neurology, BSMMU, Dhaka.

6. Dr. Mohammad Bazlur Rashid, MD (Neurology) student, Dept. of Neurology, BSMMU, Dhaka. 
During this period stroke incidence has declined by $42 \%$ in high-income countries ${ }^{5}$. Stroke is the third leading cause of death in Bangladesh, and the prevalence of stroke over age 40 is $300 /$ $100,000^{6}$. In a study of hospitalized stroke patient in Bangladesh, it was found that the incidence of ischemic stroke was $61 \%$ and hemorrhagic stroke $39 \%{ }^{7}$. Mortality due to stroke increased from $6 \%$ in 2006 to around $8.57 \%$ in year $2011^{6}$.

The etiopathogenesis of stroke is multifactorial, with multiple modifiable and non-modifiable risk factors being associated. Findings from the interstroke study suggest that HTN, current smoking, high waist-to hip ratio (abdominal obesity), sedentary lifestyle, DM, alcohol intake, psychosocial stress and depression, cardiac causes and dyslipidemia account for about $90 \%$ risk of stroke ${ }^{8}$. In a hospital based cross sectional study it was found that hyperlipidemia, DM, heart disease, obesity, cigarette smoking, oral contraception use, sedentary work, and previous history of TIA were risk factors for stroke in Bangladesh ${ }^{7}$. Various etiologies often result in different outcome, treatment and likelihood of recurrence in ischemic strokes. Large hemispheric infarcts resulting from occlusion of the internal carotid artery or proximal middle cerebral artery has the worst prognosis ${ }^{9}$. Mortality is higher among patients with large-artery atherosclerotic lesions than lacunar stroke. Recurrent strokes are more common in patients with lacunar ${ }^{10}$ and cardioembolic stroke. Anticoagulants may be prescribed to prevent recurrent cardio- embolic stroke ${ }^{11}$. Carotid stenting and carotid endarterectomy is useful in preventing recurrent stroke in patients with large-artery stenosis whereas aspirin and ticlopidine prevent recurrence in patients with small-artery occlusive disease more than large-artery stenosis ${ }^{12,13}$. In this way, determining the cause of stroke does influence the outcome and choice for management.

As the etiologies of ischemic stroke are diverse, it is difficult to include all stroke subtypes within a single classification system. The TOAST classification of subtype was introduced to produce uniformity. TOAST classification was the first classification system based on stroke mechanisms.
Vascular risk factors, early and long-term recurrence and survival were found to be different among the ischemic stroke subtypes classified by TOAST ${ }^{14,15}$.

The TOAST classification system includes five categories: 1.Large-artery atherosclerosis 2 . Cardioembolism 3. Small-vessel occlusion (lacune) 4. Stroke of other determined etiology 5. Stroke of undetermined etiology (Two or more causes identified, negative evaluation and incomplete evaluation $)^{16}$. The TOAST classification system is straightforward, valid ${ }^{17}$ and follows a logical progression ${ }^{16}$.

There is scanty data regarding ischemic stroke prevalence, associated risk factors, outcome prediction and treatment in various subtypes of ischemic stroke in our population. In order to find out causative mechanism of ischemic stroke, many investigations are needed which all are available in BSMMU, a tertiary care hospital. This study would be helpful for our doctors to know more about the ischemic stroke with their risk factors, subtypes, treatment and prevention in Bangladesh.

\section{Methods:}

This was a cross-section observational study held in department of Neurology, Bangabandhu Sheikh Mujib Medical University (BSMMU), Dhaka from January 2018 to December 2018 with the aim to explore any variations of risk factors among different subtypes of ischemic stroke according to TOAST criteria.

After ethical clearance from Institutional Review Board (IRB), this study included 52 adult patients of ischemic stroke of either sex within two weeks of first ever attack from the Department of Neurology, BSMMU. Patients with previous history of stroke, patients with hemorrhagic stroke, venous stroke and known case of malignancy, CKD and critically ill patients were excluded from this study. Purposive non random sampling was used in selecting cases.

Informed written consent was taken from each patient or from patient's attendant (for severely ill or unconscious patient). The patients were diagnosed by history, clinical examinations and 
confirmed by CT scan or MRI of Brain by a consultant neurologist.

Demographic profile including age, sex, residence, occupation, educational level, income status was recorded. Information regarding hypertension, smoking, diabetes, ischemic heart disease, valvular heart disease and other relevant history were recorded through a structured questionnaire. Height and body weight were measured to asses BMI.

The patients were assessed by diagnostic tests including cardiac imaging (echocardiography, etc.), duplex imaging of extracranial arteries, arteriography, and laboratory assessments for routine and baseline investigations like $\mathrm{CBC}$, Urine $\mathrm{RME}, \mathrm{S}$. Creatinine, Blood sugar, $\mathrm{HbA} 1 \mathrm{c}$ and Lipid profile. Special investigations like transesophgeal echocardiography, vasculitic profile, CSF study and tests for prothrombotic state were done in selected cases. For study purpose, no additional biochemical and radiological tests were done.

With all aseptic precaution, $10 \mathrm{ml}$ of blood was collected. Of them $2 \mathrm{ml}$ was collected in EDTA vial for CBC, $2 \mathrm{ml}$ in sodium citrate vial for ESR, $4 \mathrm{ml}$ in sodium citrate vial for biochemical study and $2 \mathrm{ml}$ in EDTA vial for HbA1c. Blood samples were mixed by repeated inversions for 5-7 times and then were sent to department of Laboratory Medicine with ice bag immediately for analysis. In department of Laboratory Medicine blood samples were processed further with centrifuge at 4000 RPM for 10 minutes for biochemical study. CBC was done by Hematology Autoanalyzer (Sysmex XN-2000/ $\mathrm{XT4000i)} \mathrm{and} \mathrm{was} \mathrm{re-checked} \mathrm{manually.} \mathrm{RBS,}$ HbA1c, S. Electrolyte, S. Creatinine and Lipid profile were done by Siemens Automated Biochemistry Analyzer Dimension RXL MAX, USA. Triglyceride was measured by enzymatic (end point) method, total cholesterol by cholesterol oxidase, esterase peroxidase method, LDL-C and HDL-C by direct measure (PEG) method, HbA1c by immunoturbidimetric and blood sugar by hexokinase method. Quality control (QC) was ensured by doing updated calibration and by checking 3 level QC curve showing in the autoanalyzer. Samples were stored at 2-8ÚC if there is any delay of few hours to send the samples to laboratory. All the biochemical and hematological tests were done within 14 days of index stroke. Protective measures were taken for other investigations. Hypertension, diabetes mellitus (DM), and hyperlipidemia were diagnosed according to established criteria. A structured data collection sheet was developed in English, to collect information on demographic variables, vascular risk factors, and stroke workup, and stroke subtype using TOAST criteria.

All the data were checked and edited after collection. Descriptive analysis of all relevant variables was done by using measures of Central Tendency and Dispersion. The results were expressed as means ( $\pm S D$ ) for continuous variables and as percentages for categorical variables. Data were expressed as number (percent) and managed by SPSS for Windows Version 22. Chi-square test of proportion was applied for significance of patients with risk factors. P-value of $<0.05$ was taken as statistically significant.

\section{Results:}

Mean age of the respondents was $57 \pm 12.37$ years (table1 $=$ I) with a slightly higher male predominance (Fig.I). Male to female ratio was 1.2:1. Present study showed that among the 52 ischemic stroke patients dyslipidemia $44(84.6 \%)$ and hypertension $37(71.2 \%)$ were the most common risk factors, followed by obesity and overweight 33(63.5\%), smoking 32 (61.5\%), diabetes mellitus 29 (55.8\%), family history of vascular event $27(51.9 \%$ ) and past history of vascular event 14 (26.9\%) (table II).

TOAST Subtype distribution of study population was large-artery atherosclerosis 18 (34.6\%) followed by cardioembolism 11(21.2\%), small-vessel occlusion $10(19.2 \%)$, stroke of other determined etiology $3(5.8 \%)$, and stroke of undetermined etiology $10(19.2 \%)$ of patients (table III). Large-artery atherosclerosis $18(34.6 \%)$ was the most common subtype of ischemic stroke and dyslipidemia was the most common risk factor (table IV). Distribution of cardiac abnormality among the TOAST subtypes showed that the most common cause of cardioembolic stroke in the this study was ischemic 
heart disease $(54.5 \%)$ with $P$ value 0.001 , then CRHD (45.5\%) with $P$ value $<0.001$ and DCM $(27.3 \%)$ with $P$ value 0.018 . These results were statistically significant. LAA subtype had normal ECG and Echocardiography findings (100\%) and SVO subtype had normal ECG $(90 \%)$ and normal Echocardiography findings (100\%). These results had $P$ value $<0.05$ and the results were statistically significant (table $\mathrm{V}$ ). Mean fasting serum cholesterol (total) of the respondents was $187.88 \pm 49.94 \mathrm{mg} /$ $\mathrm{dl}$, mean fasting serum HDL cholesterol was 38.51 $\pm 8.38 \mathrm{mg} / \mathrm{dl}$, mean fasting serum LDL cholesterol was $112.56 \pm 39.03 \mathrm{mg} / \mathrm{dl}$ and mean fasting serum triglyceride was $183.44 \pm 107.17 \mathrm{mg} / \mathrm{dl}$. Mean HbA1c of the respondents was $7.7 \pm 2.23 \%$. (table $\mathrm{Vl}$ ).

\section{Table-I}

Distribution of study population by age $(n=52)$

\begin{tabular}{lcc}
\hline Age group in years & $\mathrm{n}$ & $\%$ \\
\hline Less than or equal 35 years & 4 & $7.7 \%$ \\
36 to 45 years & 4 & $7.7 \%$ \\
46 to 55 years & 16 & $30.8 \%$ \\
56 to 65 years & 18 & $34.6 \%$ \\
Above 65 years & 10 & $19.2 \%$ \\
(Mean \pm SD) & $57 \pm 12.37$ \\
\hline
\end{tabular}

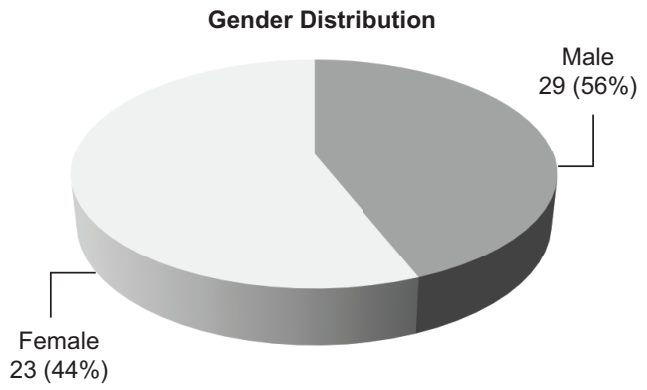

Fig.-1 (Pie chart): Frequency of study population by gender

Pie chart showing 29(56\%) of study populations were male and $23(44 \%)$ of study populations were female.

Table-II

Common Risk factors of Ischemic Stroke

\begin{tabular}{lcc}
\hline Risk factor & $\mathrm{n}$ & $\%$ \\
\hline Diabetes & 29 & $55.8 \%$ \\
Hypertension & 37 & $71.2 \%$ \\
Dyslipidemia & 44 & $84.6 \%$ \\
BMI(Over weight +Obese) & 33 & $63.5 \%$ \\
Smoking & 32 & $61.5 \%$ \\
Previous vascular event & 14 & $26.9 \%$ \\
Family history & 27 & $51.9 \%$ \\
\hline
\end{tabular}

Table-III

TOAST Subtype distribution of study population.

\begin{tabular}{lcc}
\hline TOAST Subtype & $\mathrm{n}$ & $\%$ \\
\hline Large artery atherosclerosis-LAA & 18 & $34.6 \%$ \\
Cardioembolism-CE & 11 & $21.2 \%$ \\
Small Vessel Occlusion-SVO & 10 & $19.2 \%$ \\
Stroke of other determined etiology-SODE & 3 & $5.8 \%$ \\
Stroke of undetermined etiology-SUDE & 10 & $19.2 \%$ \\
\hline Total & 52 & $100 \%$ \\
\hline
\end{tabular}

Table-IV

Risk factors among TOAST subtypes

\begin{tabular}{lccccc}
\hline & LAA & SVO & CE & SODE & SUDE \\
& $\mathrm{n}=18(\%)$ & $\mathrm{n}=10(\%)$ & $\mathrm{n}=11(\%)$ & $\mathrm{n}=3(\%)$ & $\mathrm{n}=10(\%)$ \\
\hline Diabetes & $10(55.6)$ & $6(60)$ & $5(45.5)$ & $0(0)$ & $8(80)$ \\
Hypertension & $14(77.8)$ & $7(70)$ & $6(54.5)$ & $2(66.7)$ & $8(80)$ \\
Dyslipidemia & $15(83.3)$ & $7(70)$ & $10(90.9)$ & $2(66.7)$ & $10(100)$ \\
BMI(Over weight +Obese) & $12(66.7$ & $6(60)$ & $5(45.5)$ & $2(66.7)$ & $8(80)$ \\
Smoking & $12(66.7)$ & $7(70)$ & $5(45.5)$ & $0(0)$ & $8(80)$ \\
Previous vascular event & $3(16.7)$ & $3(30)$ & $5(45.5)$ & $0(0)$ & $3(30.0)$ \\
\hline
\end{tabular}

LAA -Large artery atherosclerosis, CE- Cardioembolism, SVO- Small Vessel Occlusion, SODE- Stroke of other determined etiology, SUDE- Stroke of undetermined etiology. 
Table-V

Distribution of Cardiac Abnormality among TOAST subtypes

\begin{tabular}{llcccccc}
\hline & & \multicolumn{7}{c}{ TOAST SUBTYPE } \\
\cline { 3 - 7 } & & LAA & CE & SVO & SODE & SUDE & ${ }^{*} \mathrm{P}$ Value \\
& & $\mathrm{n}(\%)$ & $\mathrm{n}(\%)$ & $\mathrm{n}(\%)$ & $\mathrm{n}(\%)$ & $\mathrm{n}(\%)$ & \\
\hline ECG & Normal & $18(100)$ & $4(36.4)$ & $9(90)$ & $3(100)$ & $7(70)$ & $0.001^{\mathrm{s}}$ \\
& $\mathrm{MI}$ & $0(0)$ & $5(45.5)$ & $1(10)$ & $0(0)$ & $3(30)$ & $0.017^{\mathrm{s}}$ \\
& LVH & $0(0)$ & $2(18.2)$ & $0(0)$ & $0(0)$ & $0(0)$ & $0.101^{\mathrm{ns}}$ \\
& AF & $0(0)$ & $1(9.1)$ & $0(0)$ & $0(0)$ & $0(0)$ & $0.434^{\mathrm{ns}}$ \\
& Total & $18(100)$ & $11(100)$ & $10(100)$ & $3(100)$ & $10(100)$ & \\
& Normal & $18(100)$ & $0(0)$ & $10(100)$ & $3(100)$ & $7(70)$ & $<0.001^{\mathrm{s}}$ \\
& RWMA & $0(0)$ & $6(54.5)$ & $0(0)$ & $0(0)$ & $3(30)$ & $0.001^{\mathrm{s}}$ \\
& CRHD & $0(0)$ & $5(45.5)$ & $0(0)$ & $0(0)$ & $0(0)$ & $<0.001^{\mathrm{s}}$ \\
& DCM & $0(0)$ & $3(27.3)$ & $0(0)$ & $0(0)$ & $0(0)$ & $0.018^{\mathrm{s}}$ \\
& Total & $18(100)$ & $11(100)$ & $10(100)$ & $3(100)$ & $10(100)$ & \\
\hline
\end{tabular}

${ }^{*}$ Chi square test was done to measure the level of significance

$\mathrm{S}=$ significant, $\mathrm{NS}=$ not significant, $\mathrm{n}=$ Number of Patients

LAA -Large artery atherosclerosis, CE- Cardioembolism, SVO- Small Vessel Occlusion, SODE- Stroke of other determined etiology, SUDE- Stroke of undetermined etiology, MI-Myocardiac Infarction, LVH- Left Ventricular Hypertrophy, AF-Atrial Fibrillation, RWMA- Regional Wall Motion Abnormality, CRHD- Chronic Rheumatic Heart Disease, DCM- Dilated Cardiomyopathy.

Table-VI

Lipid profile and state of glycemic control of study populations

\begin{tabular}{lcc}
\hline Parameter & Number of patient & Mean \pm SD \\
\hline Total Cholesterol $(\mathrm{mg} / \mathrm{dl})$ & 52 & $187.88 \pm 49.94$ \\
HDL Cholesterol $(\mathrm{mg} / \mathrm{dl})$ & 52 & $38.51 \pm 8.38$ \\
LDL Cholesterol $(\mathrm{mg} / \mathrm{dl})$ & 52 & $112.56 \pm 39.03$ \\
Triglyceride $(\mathrm{TG})(\mathrm{mg} / \mathrm{dl})$ & 52 & $183.44 \pm 107.17$ \\
HbA1c $\%$ & 52 & $7.7 \pm 2.23$ \\
\hline
\end{tabular}

\section{Discussion:}

This study was conducted with the aim to find out the risk factors among the various subtypes of ischemic stroke according to TOAST criteria in a tertiary care hospital.

A total of 52 patients were included in the study after their first ever ischemic stroke. About $85 \%$ of patients were above 45 years. Mean age of the respondents was $57 \pm 12.37$ years. This result is consistent with a previous study in India, where mean age was $57.1( \pm 1.7)^{18}$. Another similar study of Pakistan it mean age was found to be 63 years ${ }^{19}$. These results are consistent with our study. A slightly higher male predominance was observed in this study with male to female ratio of 1.2:1. This finding was similar with the other study conducted by Saha et al., ${ }^{20}$ with a male to female ratio of 1.6:1 and by Lange et al., ${ }^{21}$ who found male to female ratio 1.5:1.The lower percentage of female stroke patients indicates either a low prevalence of stroke among females or a lower access of female stroke patients to the tertiary care hospital.

In TOAST Subtype distribution of study population, the most common subtype was large-artery atherosclerosis $18(34.6 \%)$ followed by cardioembolism 11(21.2\%), small-vessel occlusion $10(19.2 \%)$, stroke of other determined etiology 3 $(5.8 \%)$, and stroke of undetermined etiology 10 $(19.2 \%)$ of patients. In one study in Bangladesh large-artery atherosclerosis was $32.5 \%$, smallvessel occlusion was $45.3 \%$, cardioembolism was $4.8 \%$ and stroke of undetermined etiology was 
$17.2 \%$ of patients ${ }^{22}$. The number of patients in small vessel occlusion stroke was small in our study because due to less severe symptoms many patients did not reach to a tertiary care hospital like BSMMU. In India Renjen et al, ${ }^{18}$ found largeartery atherosclerosis $57.7 \%$, small-vessel occlusion $7.7 \%$, cardioembolism $4.5 \%$ and stroke of undetermined etiology $27 \%$ of patients. In this study dyslipidemia was the most common risk factor and was found in $84.6 \%$ patients. In a study in BIRDEM, Bhowmik et al., ${ }^{22}$ found dyslipidemia in $93 \%$ of patients. This finding was consistent with our study. In another study Khan et al., ${ }^{23}$ found dyslipidemia in $32.7 \%$ of patients and Renjen et al., ${ }^{18}$ found dyslipidemia in $23.7 \%$ of patients. In this study a very high proportion of the stroke patients had dyslipidemia in contrast to India and Pakistan. These differences were most likely due to change in dietary habit, sedentary life style and increasing weight of our population.

Hypertension was one of the most important modifiable risk factors which was found in 37(71.2 $\%)$ patients in this study. In a recent similar type of study Bhowmik et al., ${ }^{22}$ found that $74.2 \%$ of patients were hypertensive. Renjen et al., ${ }^{18}$ in India found it to be $56.9 \%$ and Nadia et al., ${ }^{19}$ found it to be $85 \%$. These findings are comparable to this study.

In this study 29 (55.8\%) patients had DM. Nadia et al., 2011 of Pakistan found $49 \%$ patients had DM which was similar with this study. Bhowmik et al., ${ }^{22}$ found $74.5 \%$ of patients were diabetic and the rate was higher because BIRDEM General Hospital is a tertiary care hospital in Dhaka, run by the Diabetic Association of Bangladesh.

This study found that $61.5 \%$ patients were smoker which was similar with the findings of another study in Bangladesh ${ }^{24}(61.18 \%)$. In this study, family history of vascular event was found in $51.9 \%$ patients and Bhowmik et al., ${ }^{22}$ found positive family history in $50.8 \%$ of patients which was almost similar.

Large-artery atherosclerosis was the most common subtype where most of the patients were aged over 45 years with slight male predominance. Dyslipidemia (83.3\%) and hypertension (77.8\%) were the most common risk factors, followed by smoking $(66.7 \%)$, obesity $(55.6 \%)$ and DM (55.6\%). Renjen et al., ${ }^{18}$ found Hypertension in $85 \%$ cases, Smoking in $61 \%$ cases and Diabetes in $41 \%$ cases in LAA subtype. These findings are similar with present study.

Kolominsky-Rabas et al., 25 found hypertension in $52 \%$, diabetes in $32 \%$, smoking in $25 \%$ and cardiac disease in $45 \%$ patients in LAA subtype. Cause of this difference may be due to ethnic, geographical and socioeconomic differences.

In small-vessel occlusion subtype dyslipidemia (70\%), HTN (70\%), smoking (70\%), DM (60\%), obesity $30 \%$ and overweight $30 \%$ are important risk factors with equal male to female ratio. Renjen et al., ${ }^{18}$ found coronary artery disease, hypertension, diabetes mellitus, dyslipidemia and smoking as risk factors in this group.

In our study 3 patients $(5.8 \%)$ have other determined etiology. Of them one patient was in hypercoagulable state (protein $\mathrm{C}$ and antiphospholipid antibody positive), another had neurosyphilis and other had tubercular meningitis (TBM).They were non-diabetic and non-smoker and all of them were male with positive family history of stroke. Kolominsky-Rabas et al., ${ }^{25}$ found $2 \%$ of their patients in this group and Lange et al., ${ }^{21}$ found $3.1 \%$.

In stroke of undetermined etiology subtype dyslipidemia (100\%) and hypertension $(80 \%)$ are the most common risk factors, followed by diabetes mellitus $(80 \%)$, smoking $(80 \%)$, family history of stroke $(70 \%)$, obesity $40 \%$ and overweight $40 \%$. The reason for categorizing these patients as having an undetermined etiology was two or more causes were being identified and any of which could be responsible and in a few cases evaluation was incomplete.

Distribution of cardiac abnormality among the TOAST subtypes showed that the most common cause of cardioembolic stroke in the this study was ischemic heart disease (54.5\%) with $P$ value 0.001 , then chronic rheumatic heart disease $(45.5 \%)$ with $P$ value $<0.001$ and dilated cardiomyopathy $(27.3 \%)$ with $P$ value 0.018 . These results were statistically significant. Aquil et al., ${ }^{19}$ found ischemic 
heart disease as most common risk factor whereas Renjen et al., ${ }^{18}$ found atrial fibrillation as major risk factor in CE subtype. It was also observed that -Large-artery atherosclerosis subtype had normal ECG and Echocardiography findings (100\%). Small-vessel occlusion subtype had normal ECG $(90 \%)$ and normal Echocardiography findings $(100 \%)$. These results had $P$ value $<0.05$ and the results were statistically significant.

There are several limitations of the study. The study was done in short period with small sample size. Method of sampling was purposive, i.e. nonrandom sampling and study population were enrolled from only one center hence it may not represent the whole population of the country.

\section{Conclusion:}

Present study showed that the etiopathogenesis of ischemic stroke varies among different subtypes. In this study large-artery atherosclerosis was the most common subtype, followed by cardioembolism, small vessel occlusion, stroke of undetermined etiology and stroke of other determined etiology subtypes. Dyslipidemia was found to be the most common risk factor, others were $\mathrm{HTN}$, diabetes and smoking. Although there were variations in distributions of risk factors among different subtypes, but most of them were not statistically significant. Ischemic heart disease and rheumatic heart disease were very important cause and comorbidities of cardioembolic types of ischemic stroke.

\section{Reference:}

1. Johnson, W., Onuma, O., Owolabi, M. and Sachdev, S., 2016. Stroke: a global response is needed. Bulletin of the World Health Organization, 94(9), pp.634.

2. Strong, K., Mathers, C. and Bonita, R., 2007. Preventing stroke: saving lives around the world. The Lancet Neurology, 6(2), pp.182187.

3. GBD 2016 Lifetime Risk of Stroke Collaborators, 2018. Global, regional, and country-specific lifetime risks of stroke, 1990 and 2016. New England Journal of Medicine, 379(25), pp.2429-2437.
4. Aho, K., Harmsen, P., Hatano, S., Marquardsen, J., Smirnov, V.E. and Strasser, T., 1980. Cerebrovascular disease in the community: results of a WHO collaborative study. Bulletin of the World Health Organization, 58(1), pp.113-130.

5. Feigin, V.L., Lawes, C.M., Bennett, D.A., Barker-Collo, S.L. and Parag, V., 2009. Worldwide stroke incidence and early case fatality reported in 56 population-based studies: a systematic review. The Lancet Neurology, 8(4), pp.355-369.

6. Islam, M.N., Moniruzzaman, M., Khalil, M.I., Basri, R., Alam, M.K., Loo, K.W. and Gan, S.H., 2013. Burden of stroke in Bangladesh. International journal of stroke, 8(3), pp.211-213.

7. Hossain, A.M., Ahmed, N.U., Rahman, M., Islam, M.R., Sadhya, G. and Fatema, K., 2011. Analysis of sociodemographic and clinical factors associated with hospitalized stroke patients of Bangladesh. Faridpur Medical College Journal, 6(1), pp.19-23.

8. O’Donnell, M.J., Xavier, D., Liu, L., Zhang, H., Chin, S.L., Rao-Melacini, P., Rangarajan, S., Islam, S., Pais, P., McQueen, M.J. and Mondo, C., 2010. Risk factors for ischaemic and intracerebral haemorrhagic stroke in 22 countries (the INTERSTROKE study): a casecontrol study. The Lancet, 376(9735), pp.112123.

9. Bamford, J., Sandercock, P., Dennis, M., Warlow, C. and Burn, J., 1991. Classification and natural history of clinically identifiable subtypes of cerebral infarction. The Lancet, 337(8756), pp.1521-1526.

10. Sacco, S.E., Whisnant, J.P., Broderick, J.P., Phillips, S.J. and O'Fallon, W.M., 1991. Epidemiological characteristics of lacunar infarcts in a population. Stroke, 22(10), pp.1236-1241.

11. Freeman, W.D. and Aguilar, M.I., 2011. Prevention of cardioembolic stroke. Neurotherapeutics, 8(3), pp.488. 
12. Gent, M., Easton, J.D., Hachinski, V., Panak, E., Sicurella, J., Blakely, J., Ellis, D., Harbison, J., Roberts, R., Turpie, A.G. and CATS group, 1989. The Canadian American ticlopidine study (CATS) in thromboembolic stroke. The Lancet, 333(8649), pp.1215-1220.

13. Wallaert, J.B., Cronenwett, J.L., Bertges, D.J., Schanzer, A., Nolan, B.W., De Martino, R., Eldrup-Jorgensen, J., Goodney, P.P. and Vascular Study Group of New England, 2013. Optimal selection of asymptomatic patients for carotid endarterectomy based on predicted 5 -year survival. Journal of vascular surgery, 58(1), pp.112-119.

14. Schulz, U.G.R. and Rothwell, P.M., 2003. Differences in vascular risk factors between etiological subtypes of ischemic stroke: importance of population-based studies. Stroke, 34(8), pp.2050-2059.

15. Lovett, J.K., Coull, A.J. and Rothwell, P.M., 2004. Early risk of recurrence by subtype of ischemic stroke in population-based incidence studies. Neurology, 62(4), pp.569-573.

16. Adams Jr, H.P., Bendixen, B.H., Kappelle, L.J., Biller, J., Love, B.B., Gordon, D.L. and Marsh 3rd, E.E., 1993. Classification of subtype of acute ischemic stroke. Definitions for use in a multicenter clinical trial. TOAST. Trial of Org 10172 in Acute Stroke Treatment. Stroke, 24(1), pp.35-41.

17. Meschia, J.F., Barrett, K.M., Chukwudelunzu, F., Brown, W.M., Case, L.D., Kissela, B.M., Brown Jr, R.D., Brott, T.G., Olson, T.S., Rich, S.S. and Silliman, S., 2006. Interobserver agreement in the trial of org 10172 in acute stroke treatment classification of stroke based on retrospective medical record review. Journal of Stroke and Cerebrovascular Diseases, 15(6), pp.266-272.

18. Renjen, P.N., Beg, M.A. and Ahmad, K., 2015. Epidemiological study of incidence and risk factors of Ischemic stroke subtypes according to Trial of ORG 10172 in acute stroke treatment criteria: A 3 years, hospital-based study. International Journal of Medicine and Public Health, 5(1), pp.50-54.

19. Aquil, N., Begum, I., Ahmed, A., Vohra, E.A. and Soomro, B.A., 2011. Risk factors in various subtypes of ischemic stroke according to TOAST criteria. J Coll Physicians Surg Pak, 21(5), pp.280-283.

20. Saha, R., Islam, M.S.U., Hossain, A.M., Kabir, M.R., Al Mamun, A., Saha, S.K., Mondal, S.K. and Alam, M.J., 2016. Clinical presentation and risk factors of stroke-a study of 100 hospitalized stroke patients in Bangladesh. Faridpur Medical College Journal, 11(1), pp.23-25.

21. Lange, M.C., Cabral, N.L., Moro, C.H., Longo, A.L., Gonçalves, A.R., Zétola, V.F. and Rundek, T., 2015. Incidence and mortality of ischemic stroke subtypes in Joinville, Brazil: a population-based study. Arquivos de neuropsiquiatria, 73(8), pp.648-654.

22. Bhowmik, N.B., Abbas, A., Saifuddin, M., Islam, M., Habib, R., Rahman, A., Haque, M., Hassan, Z. and Wasay, M., 2016. Ischemic strokes: Observations from a hospital based stroke registry in Bangladesh. Stroke research and treatment, 2016. pp.1-13.

23. Khan, N.I., Naz, L., Mushtaq, S., Rukh, L., Ali, S. and Hussain, Z., 2009. Ischemic stroke: prevalence of modifiable risk factors in male and female patients in Pakistan. Pakistan journal of pharmaceutical sciences, 22(1).pp.62-67.

24. Rahman, A., Aydin, H.E., Komonchan, S., Saha, U.K., Quraishi, F.A. and Hossain, S., 2014. Evaluation of modifiable risk factors for stroke in Bangladesh: A tertiary level hospital experience. International Journal of Clinical Medicine Research 1(4),pp.140-145

25. Kolominsky-Rabas, P.L., Weber, M., Gefeller, O., Neundoerfer, B. and Heuschmann, P.U., 2001. Epidemiology of ischemic stroke subtypes according to TOAST criteria: incidence, recurrence, and long-term survival in ischemic stroke subtypes: a populationbased study. Stroke, 32(12), pp.2735-2740. 